\title{
ADUBAÇÃO E PRODUÇÃO DE Paspalum EM DOIS NÍVEIS DE FERTILIDADE DE LATOSSOLO VERMELHO-AMARELO: ESTABELECIMENTO E MANUTENÇÃO ${ }^{1}$
}

\author{
Paspalum fertilizer response and yield in two fertility level of an oxisol (Hapludox): \\ establishment and maintenance
}

\author{
Odo Primavesi², Ana Cândida Primavesi ${ }^{3}$, Luiz Alberto Rocha Batista², Rodolfo Godoy²
}

\begin{abstract}
RESUMO
Foram determinadas as doses de N, $\mathrm{P}_{2} \mathrm{O}_{5}$ e $\mathrm{K}_{2} \mathrm{O}$ necessárias para a obtenção da máxima produção de forragem de Paspalum regnellii, em dois níveis de fertilidade do solo, quanto a $\mathrm{P}$ e $\mathrm{K}$. Os experimentos foram instalados em Latossolo Vermelho-Amarelo Distrófico típico, em São Carlos, SP. Utilizou-se fatorial fracionado (1/2) $4^{3}$ com dois blocos ao acaso, total de 32 parcelas, sem repetição. Os tratamentos foram quatro doses de $\mathrm{N}$ e de $\mathrm{K}_{2} \mathrm{O}\left(0,100,200\right.$ e $300 \mathrm{~kg} \mathrm{ha}^{-1}$ no primeiro e segundo ano), como uréia e cloreto de potássio respectivamente, e quatro doses de $\mathrm{P}_{2} \mathrm{O}_{5}\left(0,80,160,240 \mathrm{~kg} \mathrm{ha}^{-1}\right.$ no primeiro ano e $0,70,140$ e $210 \mathrm{~kg} \mathrm{ha}^{-1}$ no segundo ano), como superfosfato triplo. No período das chuvas, foram realizados cortes a cada 35 dias de descanso em média. As doses de N, P e K para a máxima produção de forragem de Paspalum regnellii, no primeiro ano foram, em kg ha ${ }^{-1}: \mathrm{N}=210, \mathrm{P}_{2} \mathrm{O}_{5}=240, \mathrm{~K}_{2} \mathrm{O}=190$, no solo com fertilidade baixa e $\mathrm{N}=250, \mathrm{P}_{2} \mathrm{O}_{5}=240, \mathrm{~K}_{2} \mathrm{O}=180$ no solo com fertilidade média, e no segundo ano: $\mathrm{N}=300, \mathrm{P}_{2} \mathrm{O}_{5}=210, \mathrm{~K}_{2} \mathrm{O}=300$, no solo com fertilidade baixa e $\mathrm{N}=300, \mathrm{P}_{2} \mathrm{O}_{5}=190, \mathrm{~K}_{2} \mathrm{O}=290$ no solo com fertilidade média, com produções de matéria seca de, respectivamente, $5.428 \mathrm{~kg} \mathrm{ha}^{-1} \mathrm{e} 7.789 \mathrm{~kg} \mathrm{ha}^{-1}$ no primeiro ano, e no segundo ano de $13.084 \mathrm{e} 10.468 \mathrm{~kg} \mathrm{ha}^{-1}$.
\end{abstract}

Termos para indexação: fósforo, nitrogênio, potássio, produção de forragem.

\section{ABSTRACT}

The goal of this work was to determine the best rates of $\mathrm{N}, \mathrm{P}_{2} \mathrm{O}_{5}$, and $\mathrm{K}_{2} \mathrm{O}$ to get maximum forage yield of Paspalum regnellii Bra 019186 grown in two soil fertility levels: low and medium in $\mathrm{P}$ and $\mathrm{K}$. The experiments were set up in a typical Red-yellow latosol (Hapludox), in São Carlos, SP, Brazil. Experimental design was an incomplete (1/2) $4^{3}$ factorial with a total of 32 plots, in two randomized blocks, without replication. Treatments were four rates of $\mathrm{N}$ and $\mathrm{K}_{2} \mathrm{O}\left(0,100,200\right.$, and $300 \mathrm{~kg} \mathrm{ha}^{-1}$ in the first and second year), using urea and potassium chloride, and four rates of $\mathrm{P}_{2} \mathrm{O}_{5}\left(0,80,160,240 \mathrm{~kg} \mathrm{ha}^{-1}\right.$ in the first year and 70, 140, $210 \mathrm{~kg} \mathrm{ha}^{-1}$ in the second year), using triple superphosphate. Cuttings were done, mainly each 35 days, in the rain season. The N, P, K rates for maximum yield in the first year were, in $\mathrm{kg} \mathrm{ha}^{-1}$ with low fertility: $\mathrm{N}=210, \mathrm{P}_{2} \mathrm{O}_{5}=240, \mathrm{~K}_{2} \mathrm{O}=190$; and with medium fertility: $\mathrm{N}=$ $250, \mathrm{P}_{2} \mathrm{O}_{5}=240, \mathrm{~K}_{2} \mathrm{O}=180$, and in the second year: $\mathrm{N}=300, \mathrm{P}_{2} \mathrm{O}_{5}=210, \mathrm{~K}_{2} \mathrm{O}=300$, in soil with low fertility, and $\mathrm{N}=300, \mathrm{P}_{2} \mathrm{O}_{5}$ $=190, \mathrm{~K}_{2} \mathrm{O}=290$ in soil with medium fertility, resulting in forage yields of, respectively, 5,428 and 7,789 $\mathrm{kg} \mathrm{ha}^{-1}$ of dry matter in the first year, and of 13,084 e 10,468 $\mathrm{kg} \mathrm{ha}^{-1}$ in the second year.

Index terms: forage yield, nitrogen, phosphorus, potassium.

(Recebido em 27 de abril de 2006 e aprovado em 2 de abril de 2007)

\section{INTRODUÇÃO}

Os conhecimentos obtidos por meio do estudo de respostas de plantas forrageiras à adubação podem ser instrumentos eficazes para dar suporte à escolha de um manejo mais adequado para manter a capacidade de suporte de sistemas intensivos de produção de bovinos.

Batista \& Godoy (2000) mostram a necessidade da diversificação das opções de forrageiras e a importância das espécies de Paspalum como pastagens cultivadas. $\mathrm{O}$ gênero Paspalum se destaca entre as gramíneas nativas com potencial forrageiro e resistência à cigarrinha. A fertilização com nitrogênio, fósforo e potássio pode ser um fator extremamente importante na produtividade das espécies forrageiras do gênero Paspalum, mas são escassos os trabalhos que mencionem a sua influência no aumento dessa produtividade.

Em razão da importância de novas espécies forrageiras estarem disponíveis para sistemas intensivos de produção animal e da necessidade de estudos referentes ao manejo dessas plantas e de orientação para critérios de manejo, objetivou-se, neste estudo, verificar

${ }^{1}$ Trabalho financiado pelo Convênio Embrapa/Unipasto; Forragicultura

Engenheiros Agrônomos, Doutores - Embrapa Pecuária Sudeste/CPPSE - Cx. P. 336 - 13560-970 - São Carlos, SP - odo@cppse.embrapa.br; lbatista@cppse.embrapa.br; godoy@cppse.embrapa.br

${ }^{3}$ Engenheira Agrônoma, Doutora, Aposentada - Embrapa Pecuária Sudeste/CPPSE - Cx. P. 336 - 13560-970 - São Carlos, SP - anacprima@yahoo.com.br 
a resposta da forrageira a doses crescentes de $\mathrm{N}, \mathrm{P}_{2} \mathrm{O}_{5} \mathrm{e}$ $\mathrm{K}_{2} \mathrm{O}$, e determinar as doses necessárias para obtenção da máxima produção de forragem de Paspalum regnellii $\mathrm{Mez}$ (1917), em função de fertilidade diferenciada quanto a fósforo e potássio de um Latossolo Vermelho-Amarelo, representativo da região e do ecossistema Cerrados.

\section{MATERIAL E MÉTODOS}

Os experimentos foram instalados, em Latossolo Vermelho-Amarelo Distrófico típico (LVAd), na fazenda Canchim, da Embrapa Pecuária Sudeste, situada em São Carlos, SP, latitude de $22^{\circ} 01^{\prime}$ S e longitude de $47^{\circ} 54^{\prime} \mathrm{W}$, em altitude de $836 \mathrm{~m}$, sob clima tropical de altitude. Foi coletada amostra de terra em julho de 2003 para análises químicas de rotina, e foi aplicado calcário em 18/09/2003 para elevar a saturação por bases a $60 \%$. Em 12/11/2003 foi feita nova coleta de terra da área do experimento, nas profundidades de 0-20, 20-40 e 40-60 cm para análise de rotina (RAIJ et al., 2001) e determinação do teor de nitrato (Tabela 1) e análise granulométrica (Tabela 2), e em 14/11/ 2003, adubações fosfatada e potássica para criar fertilidade média em fósforo (13 a $30 \mathrm{mg} \mathrm{dm}^{-3}$ ) e potássio (1,3 a 3,0 $\mathrm{mmol}_{\mathrm{c}} \mathrm{dm}^{-3}$ ) no solo (RAIJ et al., 1996). Em 17/11/ 2003 foram semeados $4 \mathrm{~kg} \mathrm{ha}^{-1}$ de sementes puras viáveis de Paspalum regnellii BRA 019186, em parcelas com $1 \mathrm{~m}$ de largura (cinco linhas espaçadas de $20 \mathrm{~cm}$ ) e $6 \mathrm{~m}$ de comprimento.

Utilizou-se como esquema experimental um fatorial fracionado (1/2) $4^{3}$ com dois blocos ao acaso, num total de 32 parcelas, sem repetição, de acordo com Andrade \& Noleto (1986). Os tratamentos no primeiro ano (adubação de formação) constaram de quatro doses de nitrogênio $\left(0,100,200\right.$ e $\left.300 \mathrm{~kg} \mathrm{ha}^{-1} \mathrm{de} \mathrm{N}\right)$, na forma de uréia, quatro doses de fósforo $(0,80,160,240$ $\mathrm{kg} \mathrm{ha}^{-1}$ de $\mathrm{P}_{2} \mathrm{O}_{5}$ ), como superfosfato triplo, e quatro doses de potássio $\left(0,100,200\right.$ e $300 \mathrm{~kg} \mathrm{ha}^{-1}$ de $\left.\mathrm{K}_{2} \mathrm{O}\right)$, como cloreto de potássio. No ano de estabelecimento, as doses de fósforo foram aplicadas totalmente na semeadura (17/11/03) e as de nitrogênio e potássio foram parceladas respectivamente na semeadura e após os cortes. No segundo ano (adubação de manutenção), os tratamentos constaram de quatro doses de nitrogênio $\left(0,100,200\right.$ e $\left.300 \mathrm{~kg} \mathrm{ha}^{-1} \mathrm{de} \mathrm{N}\right)$, na forma de uréia, quatro doses de $\mathrm{P}\left(0,70,140,210 \mathrm{~kg} \mathrm{ha}^{-1}\right.$ de $\mathrm{P}_{2} \mathrm{O}_{5}$ ), como superfosfato triplo, e quatro doses de potássio $\left(0,100,200\right.$ e $300 \mathrm{~kg} \mathrm{ha}^{-1}$ de $\left.\mathrm{K}_{2} \mathrm{O}\right)$, como cloreto de potássio. As doses de fósforo foram aplicadas totalmente no início da estação chuvosa, e as de nitrogênio e de potássio foram parceladas, respectivamente, no início das águas e depois de cada corte (Tabela 3).

Tabela 1 - Características químicas dos solos anteriores à instalação dos experimentos, ano 2003, e durante o andamento, em 2004.

\begin{tabular}{|c|c|c|c|c|c|c|c|c|c|c|}
\hline $\begin{array}{c}\text { Prof. } \\
\mathrm{cm}\end{array}$ & $\begin{array}{c}\mathrm{pH} \\
\mathrm{CaCl}_{2}\end{array}$ & $\begin{array}{c}\mathrm{MO} \\
\mathrm{g} \mathrm{dm}^{-3}\end{array}$ & $\begin{array}{c}\mathrm{P} \\
\mathrm{mg} \mathrm{dm}^{-3}\end{array}$ & $\mathrm{~K}$ & $\mathrm{Ca}$ & $\begin{array}{l}\mathrm{Mg} \\
-\mathrm{mm}\end{array}$ & $\begin{array}{l}\mathrm{H}+\mathrm{Al} \\
\mathrm{m}^{-3}---\end{array}$ & CTC & $\begin{array}{l}\mathrm{V} \\
\%\end{array}$ & $\begin{array}{l}\mathrm{N}-\mathrm{NO}_{3}{ }^{-} \\
\mathrm{mg} \mathrm{dm}^{-3}\end{array}$ \\
\hline \multicolumn{11}{|c|}{ Amostragem em 12/11/2003* } \\
\hline \multicolumn{11}{|c|}{ Fertilidade baixa } \\
\hline $0-20$ & 5,1 & 16 & 4 & 1,0 & 19 & 10 & 24 & 54 & 55 & 12,6 \\
\hline $20-40$ & 4,8 & 13 & 2 & 0,6 & 12 & 5 & 23 & 41 & 43 & 3,7 \\
\hline $40-60$ & 4,8 & 12 & 2 & 0,5 & 10 & 4 & 22 & 37 & 39 & 3,9 \\
\hline \multicolumn{11}{|c|}{ Fertilidade média } \\
\hline $0-20$ & 5,1 & 17 & 4 & 0,9 & 18 & 10 & 22 & 51 & 56 & 7,8 \\
\hline $20-40$ & 4,8 & 13 & 3 & 0,6 & 13 & 5 & 24 & 43 & 44 & 9,8 \\
\hline $40-60$ & 4,8 & 12 & 2 & 0,4 & 9 & 3 & 23 & 36 & 35 & 4,4 \\
\hline \multirow{2}{*}{\multicolumn{11}{|c|}{$\begin{array}{c}\text { Amostragem em 12/03/2004 } \\
\text { Fertilidade baixa }\end{array}$}} \\
\hline & & & & & & & & & & \\
\hline $0-20$ & 4,7 & 12 & 1 & 0,6 & 11 & 8 & 30 & 49 & 40 & - \\
\hline \multicolumn{11}{|c|}{ Fertilidade média } \\
\hline $0-20$ & 5,1 & 14 & 15 & 0,9 & 16 & 9 & 25 & 51 & 51 & - \\
\hline
\end{tabular}

Prof.= profundidade. $*$ Calagem realizada em 18/09/2003. Adubação corretiva de $\mathrm{P}_{2} \mathrm{O}_{5}$ e $\mathrm{K}_{2} \mathrm{O}$ para nível de fertilidade média em 14/ 12/2003. Adubação de semeadura em 17/11/2003. 
Tabela 2 - Granulometria do solo.

\begin{tabular}{|c|c|c|c|}
\hline Profundidade & Areia & Argila & Silte \\
\hline $\mathrm{cm}$ & -------------. & $\mathrm{g} \mathrm{kg}^{-1}$ & ------------- \\
\hline $0-20$ & 732 & 238 & 30 \\
\hline
\end{tabular}

Tabela 3 - Parcelamento das doses dos adubos contendo nitrogênio e potássio.

\begin{tabular}{|c|c|c|c|c|}
\hline \multirow[t]{2}{*}{ Doses totais de $\mathrm{N}$ e $\mathrm{K}_{2} \mathrm{O}$} & \multicolumn{4}{|c|}{ Época de aplicação, no primeiro e segundo ano, de doses de $\mathrm{N}_{\text {e }} \mathrm{K}_{2} \mathrm{O}$} \\
\hline & $\begin{array}{c}\text { Plantio/ Início das } \\
\text { águas }\end{array}$ & $\begin{array}{l}\text { Após primeiro } \\
\text { corte }\end{array}$ & $\begin{array}{l}\text { Após segundo } \\
\text { corte }\end{array}$ & $\begin{array}{l}\text { Após terceiro } \\
\text { corte }\end{array}$ \\
\hline $\mathrm{kg} \mathrm{ha}^{-1}$ & \multicolumn{4}{|c|}{$\mathrm{kg} \mathrm{ha}^{-1}$} \\
\hline 100 & 20 & 35 & 35 & 10 \\
\hline 200 & 40 & 70 & 70 & 20 \\
\hline 300 & 40 & 100 & 100 & 60 \\
\hline
\end{tabular}

Primeiro ano (primeiro corte: 19/03/2004; segundo corte: 19/04/2004; terceiro corte: 14/06/2004).

Segundo ano (primeiro corte: 13/12/2004; segundo corte: 17/01/2005; terceiro corte: 21/02/2005; quarto corte: 14/06/2005).

A precipitação no período estudado foi de 1.012 $\mathrm{mm}$ no primeiro ano, e de $999 \mathrm{~mm}$ no segundo ano.

A área das parcelas era de $6 \mathrm{~m}^{2}$, e a produção de forragem foi em área útil de $3 \mathrm{~m}^{2}$. As plantas foram cortadas a uma altura de $15 \mathrm{~cm}$, a cada 35 dias de descanso, no período das chuvas. Após os cortes foi feita a pesagem da matéria fresca da parcela, e retirada uma amostra com $500 \mathrm{~g}$ que foi secada a $60^{\circ} \mathrm{C}$ em estufa com circulação forçada de ar por 48 horas até peso constante, para a determinação do teor de água e posterior cálculo da matéria seca. A partir de alíquotas de matéria seca, foi determinado o conteúdo de fibra não solúvel, em detergente neutro (FDN) utilizando o método descrito por Souza et al. (1999), e de proteína bruta (PB) conforme método descrito por Silva (1981).

Os dados obtidos foram submetidos à análise de regressão e de variância, utilizando-se, respectivamente, os procedimentos REG e GLM do pacote estatístico SAS Institute (1993). Para as produções de cada experimento, por meio do modelo polinomial quadrático, foram ajustadas funções da superfície de resposta do tipo $\mathrm{Y}=\mathrm{b}_{0}+\mathrm{b}_{1} \mathrm{~N}+$ $\mathrm{b}_{2} \mathrm{~N}^{2}+\mathrm{b}_{3} \mathrm{P}+\mathrm{b}_{4} \mathrm{P}^{2}+\mathrm{b}_{5} \mathrm{~K}+\mathrm{b}_{6} \mathrm{~K}^{2}+\mathrm{b}_{7} \mathrm{NP}+\mathrm{b}_{8} \mathrm{NK}+\mathrm{b}_{9} \mathrm{PK}$, onde Y é a produção de matéria seca de forragem $\left(\mathrm{t} \mathrm{ha}^{-1}\right)$, b é o coeficiente de regressão, e N, P e K são as doses de N, $\mathrm{P}_{2} \mathrm{O}_{5}$ e $\mathrm{K}_{2} \mathrm{O}$, em kg ha ${ }^{-1}$, respectivamente.

\section{RESULTADOS E DISCUSSÃO}

\section{Primeiro ano}

A análise de variância detectou, no solo com fertilidade baixa em $\mathrm{P}$ e $\mathrm{K}$, resposta ao nitrogênio e ao fósforo e, no solo com fertilidade média, apenas ao nitrogênio (Tabela 4) para a produção de forragem. Foi verificada resposta quadrática ao nitrogênio nos dois solos, o que era esperado, pois a análise de $\mathrm{N}$-nítrico no início do experimento, nos dois solos, indicou média disponibilidade de nitrogênio. Valores acima de $20 \mathrm{mg} \mathrm{dm}^{-3}$ na camada superior (120 a $140 \mathrm{mg} \mathrm{dm}^{-3}$ no perfil do solo de $100 \mathrm{~cm}$ ) são considerados adequados para elevada produção de grãos (BUCHNER \& STURM, 1980), teor a partir do qual não ocorre resposta à adubação nitrogenada (Heitor Cantarella, $2006)^{4}$.

Os teores de proteína bruta apresentaram resposta linear às doses de nitrogênio, e variaram de 12,7\% a 17,3\% dependendo do tratamento, no solo com fertilidade baixa e de $13 \%$ a $17,3 \%$ no solo com fertilidade média. Esses teores são considerados excelentes, pois o valor do nível crítico de proteína bruta na dieta, estabelecido por Milford \& Minson (1965), como limite do consumo voluntário, é de $7 \%$. Porém, deve-se considerar que a resposta ao $\mathrm{N}$ foi quadrática, sugerindo que os valores mais elevados de $\mathrm{PB}$, na realidade, refletem acúmulo de formas não protéicas ou mesmo mineral de $\mathrm{N}$ na forragem, como o $\mathrm{N}$-nítrico, parecendo estar o máximo de proteína real em torno de 14\% +/- 2\% (PRIMAVESI et al., 2001, 2003).

Houve resposta linear dos teores de FDN às doses de nitrogênio e de potássio no solo com fertilidade baixa, o que não foi observado no solo com fertilidade média em $\mathrm{P}$ e K. Os teores de FDN variaram de $68,7 \%$ a $72,9 \%$ no solo com fertilidade baixa, e de $71,9 \%$ a $75,2 \%$ no solo com

${ }^{4}$ Comunicação pessoal, Instituto Agronômico de Campinas. 
fertilidade média. O conteúdo de FDN de uma forragem é importante tanto para avaliação da sua qualidade nutricional como também por estar relacionada ao consumo máximo de matéria seca (MERTENS, 1994). Para Sandles (1999), quando o teor de FDN aumenta a ingestão de matéria seca reduz, e segundo Brâncio et al. (2002), quando os valores de FDN nas folhas e colmos de três cultivares de Panicum maximum Jacq. foram superiores a $75 \%$, houve redução do consumo de matéria seca nessas pastagens, mesmo considerando-se uma dieta composta exclusivamente por folhas. Os valores de FDN, obtidos no presente trabalho, são consistentes com os observados na literatura para capins tropicais (REID et al., 1988; SILVA et al., 2002).

Na Tabela 5, observa-se a produção de forragem de $P$. regnellii, nos solos com baixa e média fertilidade, no primeiro ano de estabelecimento.

No solo com fertilidade baixa, as produções de forragem aumentaram até a dose de $200 \mathrm{~kg} \mathrm{ha}^{-1}$ de $\mathrm{N}$, enquanto no solo com fertilidade média os dados indicam proximidade do ponto de máxima acumulação, com a dose de $300 \mathrm{~kg} \mathrm{ha}^{-1}$ de $\mathrm{N}$. Nesse solo não houve diferença entre as doses 100, 200 e $300 \mathrm{~kg} \mathrm{ha}^{-1}$ de $\mathrm{N}$ na produção de matéria seca total de forragem, o mesmo sendo observado no solo com fertilidade média, mas em ambos os solos os tratamentos com nitrogênio resultaram em maiores produções de forragem que o tratamento que não recebeu nitrogênio. Essas maiores produções totais de forragem nos tratamentos que receberam nitrogênio, em relação aos que não receberam, também foi verificada por Kalmbacher \& Martin (1999) em Paspalum atratum Swallen. Somente no solo com fertilidade baixa houve resposta ao fósforo, sendo a maior produção total de forragem obtida com a dose de $240 \mathrm{~kg} \mathrm{ha}^{-1}$ de $\mathrm{P}_{2} \mathrm{O}_{5}$.

No solo com fertilidade baixa houve efeito das doses de fósforo na produção de forragem no primeiro e segundo cortes, e das doses de nitrogênio no segundo corte. No solo com fertilidade média houve resposta apenas às doses de nitrogênio no segundo e terceiro cortes. $\mathrm{O}$ solo com fertilidade baixa apresentava teores baixos de P-resina (Tabela 1), provavelmente por este motivo houve resposta à aplicação desse nutriente. A faixa de valores baixos para concentração de P-resina para culturas perenes é de 6 a 12 $\mathrm{mg} \mathrm{dm}{ }^{-3}$ e de valores médios é de 13 a $30 \mathrm{mg} \mathrm{dm}^{-3}$, conforme Raij et al. (1996).

As produtividades de forragem obtidas no solo com fertilidade média em $\mathrm{P}$ e $\mathrm{K}$, foram maiores que as obtidas no solo com fertilidade baixa. Sabe-se que são esperadas altas respostas ao nitrogênio em solos corrigidos e com alta ou média disponibilidade de fósforo e potássio (CANTARELLA, 1996), provavelmente devido a um melhor desenvolvimento radicular das plantas, que encontram maiores quantidades de nutrientes disponíveis.

Na Tabela 5 também encontram-se os teores médios, em dois cortes, de proteína bruta $(\mathrm{PB})$ e de fibra detergente neutro (FDN). Nos dois solos os teores de proteína aumentaram somente com as doses de nitrogênio.

Esse aumento do teor de proteína com o aumento das doses de $\mathrm{N}$ é esperado, pois o $\mathrm{N}$ absorvido pelas plantas junta-se às cadeias carbonadas formando os aminoácidos que aumentam o teor de proteína bruta da forragem (NOLLER \& RHYKERD, 1974). Outros autores também verificaram esse efeito benéfico do nitrogênio na composição química da forragem produzida (COSTA et al., 1992; RUGGIERI et al., 1995).

Embora não tenha havido interação entre os nutrientes, verificou-se (Tabela 6) que nos solos com fertilidade baixa e média, com a dose de $80 \mathrm{~kg} \mathrm{ha}^{-1} \mathrm{de}_{2} \mathrm{O}_{5}$ houve aumento da produção de matéria seca, com o acréscimo das doses de nitrogênio. Já nas doses 160 e 240 $\mathrm{kg} \mathrm{ha}^{-1}$ de $\mathrm{P}_{2} \mathrm{O}_{5}$, as maiores produções de forragem foram alcançadas com a dose de $200 \mathrm{~kg} \mathrm{ha}^{-1}$ de nitrogênio, em ambos os solos, sendo o melhor tratamento o que recebeu $240 \mathrm{~kg} \mathrm{ha}^{-1}$ de $\mathrm{P}_{2} \mathrm{O}_{5}$ e $200 \mathrm{~kg} \mathrm{ha}^{-1}$ de nitrogênio.

Tabela 4 - Média da produção de forragem, de Paspalum regnellii e média dos teores de proteína bruta e de FDN, em resposta à adubação com $\mathrm{N}, \mathrm{P}_{2} \mathrm{O}_{5}$ e $\mathrm{K}_{2} \mathrm{O}$ e em duas fertilidades de solo, no primeiro ano de plantio.

\begin{tabular}{|c|c|c|c|c|c|c|}
\hline \multirow[t]{2}{*}{ Estatísticas } & \multicolumn{3}{|c|}{ Solo fertilidade baixa } & \multicolumn{3}{|c|}{ Solo fertilidade média } \\
\hline & $\begin{array}{c}\text { Matéria seca } \\
\mathrm{kg} \mathrm{ha}^{-1}\end{array}$ & $\begin{array}{c}\mathrm{PB} \\
\%\end{array}$ & $\begin{array}{c}\text { FDN } \\
\%\end{array}$ & $\begin{array}{c}\text { Matéria seca } \\
\mathrm{kg} \mathrm{ha}^{-1}\end{array}$ & $\begin{array}{c}\mathrm{PB} \\
\%\end{array}$ & $\begin{array}{c}\text { FDN } \\
\%\end{array}$ \\
\hline Média & 3.100 & 15,0 & 71,7 & 5.542 & 15,0 & 72,9 \\
\hline Teste F & $\begin{array}{c}\mathrm{NL}^{* *} \\
\mathrm{NQ}^{* *}, \mathrm{PL}^{* *}\end{array}$ & $\mathrm{NL} * *$ & $\begin{array}{l}\mathrm{NL}^{*}, \mathrm{KL}^{*}, \\
\mathrm{NKL}^{*}\end{array}$ & $\mathrm{NL}^{* *}, \mathrm{NQ}^{* *}$ & $\begin{array}{l}\text { NL**, } \\
\text { NKL*** }\end{array}$ & ns \\
\hline $\mathrm{CV}, \%$ & 34,4 & 4,0 & 1,4 & 20,1 & 4,6 & 3,2 \\
\hline$r^{2}$ & 0,77 & 0,86 & 0,64 & 0,78 & 0,79 & 0,29 \\
\hline
\end{tabular}

Em teste F são indicados somente os coeficientes significativos ao nível de $5(*)$ e $1 \%$ (**).

$\mathrm{NL}, \mathrm{NQ}=$ componente linear e quadrático para $\mathrm{N}$, respectivamente. $\mathrm{PL}=$ componente linear para $\mathrm{P}$ 
Tabela 5 - Produção de forragem de Paspalum regnellii e teores de proteína bruta (PB) e de fibra em detergente neutro $(\mathrm{FDN})$, em resposta à adubação com $\mathrm{N}, \mathrm{P}_{2} \mathrm{O}_{5}$ e $\mathrm{K}_{2} \mathrm{O}$, em duas fertilidades de solo, no primeiro ano de estabelecimento.

\begin{tabular}{|c|c|c|c|c|c|c|}
\hline \multirow[t]{2}{*}{ Doses } & \multicolumn{3}{|c|}{ Solo com fertilidade baixa } & \multicolumn{3}{|c|}{ Solo com fertilidade média } \\
\hline & Produção & FDN & PB & Produção & FDN & PB \\
\hline & $\mathrm{kg} \mathrm{ha}^{-1} \mathrm{MS}$ & ---------- & -------- & $\mathrm{kg} \mathrm{ha}^{-1} \mathrm{MS}$ & -------- & 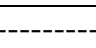 \\
\hline \multicolumn{7}{|c|}{ 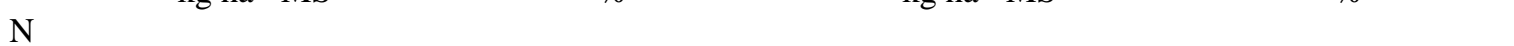 } \\
\hline 0 & 1632 & 70,9 & 13,8 & 3063 & 72,3 & 14,0 \\
\hline 100 & 3367 & 71,6 & 14,6 & 5745 & 73,2 & 14,4 \\
\hline 200 & 4026 & 72,5 & 15,2 & 6612 & 72,3 & 15,1 \\
\hline 300 & 3321 & 71,8 & 16,8 & 6750 & 73,6 & 16,3 \\
\hline Dms & $1694 *$ & $0,6 * *$ & $1,6 * *$ & $2624 *$ & Ns & $1,4 *$ \\
\hline \multicolumn{7}{|l|}{$\mathrm{P}$} \\
\hline 0 & 1471 & 71,7 & 15,1 & 5206 & 73,2 & 15,3 \\
\hline 80 & 3382 & 71,6 & 15,5 & 5551 & 73,2 & 15,0 \\
\hline 140 & 3228 & 72,1 & 14,6 & 5450 & 72,0 & 15,0 \\
\hline 210 & 4265 & 71,4 & 15,2 & 5963 & 73,1 & 14,6 \\
\hline Dms & $1694 *$ & $\mathrm{~ns}(7 \%)$ & $\mathrm{ns}$ & $\mathrm{ns}$ & Ns & $\mathrm{ns}$ \\
\hline \multicolumn{7}{|l|}{$\mathrm{K}$} \\
\hline 0 & 2398 & 71,0 & 15,2 & 4978 & 72,6 & 15,2 \\
\hline 100 & 3645 & 71,3 & 15,4 & 5930 & 72,5 & 15,1 \\
\hline 200 & 2957 & 72,4 & 14,8 & 5868 & 72,8 & 14,7 \\
\hline 300 & 3345 & 72,1 & 15,0 & 5394 & 73,3 & 14,8 \\
\hline Dms & $\mathrm{ns}$ & $0,6^{* *}$ & ns & ns & Ns & $\mathrm{ns}$ \\
\hline CV\% & 26,4 & 0,4 & 5,0 & 22,8 & 3,7 & 4,4 \\
\hline$r^{2}$ & 0,98 & 0,99 & 0,97 & 0,95 & 0,83 & 0,97 \\
\hline
\end{tabular}

Tabela 6 - Produção de matéria seca (MS) de forragem de Paspalum regnellii para as doses de nitrogênio interagindo com as de fósforo em solos de fertilidade baixa e média, no primeiro ano de estabelecimento.

\begin{tabular}{ccccccccc}
\hline $\begin{array}{c}\text { Doses de } \\
\mathrm{P}_{2} \mathrm{O}_{5} \\
\mathrm{~kg} \mathrm{ha}^{-1}\end{array}$ & \multicolumn{3}{c}{ Solo com fertilidade baixa } & \multicolumn{3}{c}{ Solo com fertilidade média } \\
& 0 & 100 & 200 & 300 & 0 & 100 & 200 & 300 \\
\hline 0 & 757 & 1.729 & 2.132 & 1.266 & 2.095 & 6.206 & 6.014 & 6.510 \\
80 & 1.959 & 3602 & 3.514 & 4.454 & 3.683 & 5.627 & 5.538 & 7.355 \\
160 & 1.760 & 3.335 & 4.352 & 3.466 & 3.600 & 5.542 & 6.411 & 6.245 \\
240 & 2.053 & 4.803 & 6.106 & 4.096 & 2.873 & 5.603 & 8.484 & 6.891 \\
\hline
\end{tabular}

No primeiro ano, as equações relacionando produção de forragem e doses de $\mathrm{N}, \mathrm{P}_{2} \mathrm{O}_{5}$ e $\mathrm{K}_{2} \mathrm{O}$ aplicadas nos dois experimentos, com indicação de significância dos coeficientes ( $* *$ nível de $1 \%$ ), foram: $\mathrm{Y}=-421,39777+$ $25,3411 * * \mathrm{~N}-0,06101 * * \mathrm{~N}^{2}+14,37363 * * \mathrm{P}-0,03415 \mathrm{P}^{2}+$ $11,20639 \mathrm{~K}-0,02147 \mathrm{~K}^{2}+0,01905 \mathrm{NP}-0,02408 \mathrm{NK}+$ 0,00832PK, no solo de fertilidade baixa, e Y1 $=2.568,697+$ $29,18256 * * \mathrm{~N}-0,06358 * * \mathrm{~N}^{2}+0,50528 \mathrm{P}+0,00658 \mathrm{P}^{2}-$
$10,61541 \mathrm{~K}-0,03565 \mathrm{~K}^{2}+0,0044 \mathrm{NP}+0,00863 \mathrm{NK}-$ 0,00023511PK, no solo de fertilidade média.

As doses de fertilizantes para a máxima produção agronômica de forragem de $P$. regnelli, no período chuvoso, foram de $210 \mathrm{~kg} \mathrm{ha}^{-1}$ de $\mathrm{N}, 240 \mathrm{~kg} \mathrm{ha}^{-1}$ de $\mathrm{P}_{2} \mathrm{O}_{5}$ e de $190 \mathrm{~kg}$ $\mathrm{ha}^{-1}$ de $\mathrm{K}_{2} \mathrm{O}$ no solo com fertilidade baixa; e no solo com fertilidade média de $250 \mathrm{~kg} \mathrm{ha}^{-1}$ de $\mathrm{N}, 240 \mathrm{~kg} \mathrm{ha}^{-1} \mathrm{de}_{2} \mathrm{O}_{5} \mathrm{e}$ de $180 \mathrm{~kg} \mathrm{ha}^{-1}$ de $\mathrm{K}_{2} \mathrm{O}$. As produções máximas foram de 
5.428 e $7.789 \mathrm{~kg} \mathrm{ha}^{-1}$, respectivamente, nos solos com fertilidade baixa e média.

Essas doses de nutrientes para adubação de formação de gramíneas são bem maiores do que as recomendadas para pasto exclusivo do grupo II no Estado de São Paulo, grupo no qual o gênero Paspalum está incluído, e cujas doses para solos com fertilidade baixa são para $\mathrm{N}, \mathrm{P}_{2} \mathrm{O}_{5}$ e $\mathrm{K}_{2} \mathrm{O}: 0,80$ e $50 \mathrm{~kg} \mathrm{ha}^{-1}$ e para fertilidade média: 0,40 e 0 , respectivamente. Se 30 dias após a germinação, as plantas apresentam sintomas de deficiência de nitrogênio aplicam-se $40 \mathrm{~kg} \mathrm{ha}^{-1}$ de N (WERNER et al., 1996). No entanto, deve-se considerar que esses valores são recomendados para as forrageiras manejadas em sistema extensivo de pastejo.

\section{Segundo ano}

A análise de variância, detectou que no segundo ano, no solo com fertilidade baixa em $\mathrm{P}$ e $\mathrm{K}$ houve resposta ao nitrogênio e ao fósforo, com interação $\mathrm{N} \mathrm{x} \mathrm{P}$ significativa, e no solo com fertilidade média apenas ao nitrogênio (Tabela 7).

A produtividade de forragem obtida no solo com fertilidade média, foi semelhante à obtida no solo com fertilidade baixa, indicando que, provavelmente, no segundo ano, a fertilidade do solo deixa de ter influência na resposta à adubação. Em trabalhos de adubação para recuperar pastagens degradadas tem-se observado maiores produções de forragem no segundo ano, em relação ao primeiro (CUNHA et al., 2001; OLIVEIRA et al., 2003, 2005)

Tabela 7 - Produção de matéria seca (MS) de Paspalum regnellii, em duas fertilidades de solo, em resposta à adubação com $\mathrm{N}, \mathrm{P}_{2} \mathrm{O}_{5}$ e $\mathrm{K}_{2} \mathrm{O}$, no segundo ano.

\begin{tabular}{|c|c|c|c|c|c|c|c|c|c|c|}
\hline \multirow[t]{3}{*}{ Doses } & \multicolumn{10}{|c|}{ Produção de forragem } \\
\hline & \multicolumn{5}{|c|}{------- Cortes - Fertilidade baixa -------- } & \multicolumn{5}{|c|}{--- Cortes - Fertilidade média -------- } \\
\hline & 1 & 2 & 3 & 4 & total & 1 & 2 & 3 & 4 & total \\
\hline $\mathrm{kg} \mathrm{ha}^{-1}$ & & & & & & $\mathrm{~g} \mathrm{ha}^{-1} \mathrm{M}$ & & & & \\
\hline \multicolumn{11}{|l|}{$\mathrm{N}$} \\
\hline 0 & 1.618 & 2.236 & 924 & 1.282 & 6.059 & 1.763 & 1.452 & 785 & 817 & 4.817 \\
\hline 100 & 1.883 & 2.821 & 1.233 & 1.294 & 7.230 & 2.273 & 2.255 & 983 & 1.347 & 6.859 \\
\hline 200 & 2.435 & 3.550 & 1.090 & 1.737 & 8.812 & 2.601 & 2.259 & 1.016 & 1.738 & 7.614 \\
\hline 300 & 2.198 & 2.950 & 1.155 & 1.866 & 8.168 & 3.063 & 2.953 & 1.207 & 1.866 & 9.089 \\
\hline Dms & ns & $\mathrm{ns}$ & $\mathrm{ns}$ & ns & ns & $890 *$ & $818 *$ & ns & $846^{*}$ & $1901 * *$ \\
\hline \multicolumn{11}{|l|}{$\mathrm{P}_{2} \mathrm{O}_{5}$} \\
\hline 0 & 1.277 & 1.867 & 833 & 934 & 4.912 & 2.374 & 2.076 & 1.050 & 1.438 & 6.938 \\
\hline 70 & 2.093 & 2.889 & 1.172 & 1.519 & 7.673 & 2.401 & 2.311 & 1.112 & 1.490 & 7.313 \\
\hline 140 & 2.144 & 2.909 & 1.427 & 1.550 & 8.030 & 2.512 & 2.343 & 909 & 1.400 & 7.165 \\
\hline 210 & 2.620 & 3.891 & 969 & 2.176 & 9.655 & 2.413 & 2.189 & 920 & 1.440 & 6.962 \\
\hline Dms & ns & $\mathrm{ns}$ & 501 & ns & ns $(7 \%)$ & Ns & ns & ns & ns & ns \\
\hline \multicolumn{11}{|l|}{$\mathrm{K}_{2} \mathrm{O}$} \\
\hline 0 & 1.867 & 2.723 & 1.234 & 1.187 & 7.010 & 2.249 & 2.225 & 965 & 1.187 & 6.625 \\
\hline 100 & 2.347 & 3.397 & 1.091 & 1.450 & 8.285 & 2.568 & 2.265 & 923 & 1.422 & 7.177 \\
\hline 200 & 1.798 & 2.559 & 1.028 & 1.719 & 7.105 & 2.488 & 2.382 & 1.059 & 1.519 & 7.448 \\
\hline 300 & 2.121 & 2.877 & 1.048 & 1.823 & 7.869 & 2.396 & 2.047 & 1.044 & 1.641 & 7.128 \\
\hline Dms & ns & ns & ns & $\mathrm{ns}$ & $\mathrm{ns}$ & Ns & ns & ns & ns & ns \\
\hline Média & & & & & 7.567 & & & & & 7.095 \\
\hline Teste F & & & & $\mathrm{NL}^{*}, \mathrm{PI}$ & $* *, \mathrm{NPL}^{*}$ & & & & & NL** \\
\hline CV\% & & & & & 31,1 & & & & & 12,9 \\
\hline $\mathrm{r}^{2}$ & & & & & 0,93 & & & & & 0,98 \\
\hline
\end{tabular}

Em teste $\mathrm{F}$ são indicados somente os coeficientes significativos ao nível de 5 (*) e $1 \%$ (**).

$\mathrm{NL}=$ componente linear e quadrático para $\mathrm{N}$, respectivamente. $\mathrm{PL}=$ componente linear para $\mathrm{P} ; \mathrm{NP}=$ interação $\mathrm{N} \times \mathrm{P}$. 
e este fato pode estar relacionado à necessidade de recuperação das estruturas da planta forrageira, como a coroa e o sistema radicular para que a mesma possa expressar seu potencial de resposta em produtividade (OLIVEIRA et al., 2003).

No presente experimento, no solo com fertilidade baixa, onde as quantidades de nutrientes disponíveis são pequenas, é possível que as plantas em formação não apresentassem, no primeiro ano, a coroa e o sistema radicular bem desenvolvidos, não tendo assim a possibilidade de atingir seu potencial de produtividade. No segundo ano, as produções de forragem foram maiores que as obtidas no primeiro ano, em especial na baixa fertilidade, provavelmente porque na fase de estabelecimento, a demanda de nutrientes pelas plantas era maior, por estarem crescendo, formando e desenvolvendo seus órgãos, e no segundo ano (manutenção), a biomassa da coroa já se formou e o sistema radicular já desenvolvido absorvia nutrientes e água de volume bem maior de solo.

Na Tabela 7 observam-se a produção de forragem de $P$. regnellii por corte e total, nos solos com baixa e média fertilidade, no segundo ano.

No solo com fertilidade média, a produção de forragem aumentou até a dose de $300 \mathrm{~kg} \mathrm{ha}^{-1}$ de nitrogênio. Nesse solo não houve diferença entre as doses 100, $200 \mathrm{e}$ $300 \mathrm{~kg} \mathrm{ha}^{-1}$ de nitrogênio na produção de matéria seca total de forragem, mas os tratamentos com nitrogênio resultaram em maiores produções de forragem que o tratamento que não recebeu nitrogênio. Somente no solo com fertilidade baixa houve resposta ao fósforo, sendo a maior produção total de forragem obtida com a dose de $210 \mathrm{~kg} \mathrm{ha}^{-1}$ de $\mathrm{P}_{2} \mathrm{O}_{5}$.

No segundo ano, as maiores produções de matéria seca de forragem foram obtidas no segundo corte, no solo com fertilidade baixa e no primeiro corte, no solo com fertilidade média. No segundo ano, no solo com baixa fertilidade em P e K o fósforo foi o nutriente mais limitante na produção de forragem, seguido do nitrogênio, e no solo com fertilidade média, somente o nitrogênio. No solo com fertilidade baixa houve efeito de doses de fósforo na produção de forragem, no terceiro corte. No solo com fertilidade média houve resposta ao nitrogênio no primeiro, segundo e quarto cortes.

Verificou-se interação N x P (Tabela 7) no solo com fertilidade baixa, sendo que com a dose de $140 \mathrm{~kg} \mathrm{ha}^{-1} \mathrm{de}$ $\mathrm{P}_{2} \mathrm{O}_{5}$ houve aumento da produção de matéria seca, com o acréscimo das doses de nitrogênio e nas doses de 70 e 210 $\mathrm{kg} \mathrm{ha}^{-1}$ de $\mathrm{P}_{2} \mathrm{O}_{5}$ as maiores produções de forragem foram alcançadas com a dose de $200 \mathrm{~kg} \mathrm{ha}^{-1}$ de N (Tabela 8). No solo com fertilidade média, houve aumento da produção de matéria seca, com o acréscimo das doses de nitrogênio para todas as doses de fósforo.

No segundo ano, as equações relacionando produção de forragem de Paspalum e doses de $\mathrm{N}, \mathrm{P}_{2} \mathrm{O}_{5} \mathrm{e}$ $\mathrm{K}_{2} \mathrm{O}$ aplicadas nos dois experimentos, com indicação de significância dos coeficientes ( $* *$ no nível de 1\%), foram: $\mathrm{Y}=5.917,273+2,927 \mathrm{~N}-0,04539 \mathrm{~N}^{2}+17,45193 \mathrm{P}-0,05796 \mathrm{P}^{2}$ $-2,60563 \mathrm{~K}-0,01277 \mathrm{~K}^{2}+0,10312 * * \mathrm{NP}+0,0518 \mathrm{NK}+$ 0,00057735PK, no solo de fertilidade baixa, e Y1 $=5768,19+$ $12,0652 \mathrm{~N}-0,01417 \mathrm{~N}^{2}-1,47136 \mathrm{P}-0,02947 \mathrm{P}^{2}-0,21993 \mathrm{~K}-$ $0,02181 \mathrm{~K}^{2}+0,01189 \mathrm{NP}+0,03004 \mathrm{NK}+0,03844 \mathrm{PK}$, no solo de fertilidade média.

As doses de fertilizantes, para a máxima produção agronômica de forragem de $P$. regnelli, foram de $300 \mathrm{~kg} \mathrm{ha}^{-1}$ de N, $210 \mathrm{~kg} \mathrm{ha}^{-1}$ de $\mathrm{P}_{2} \mathrm{O}_{5}$ e de $300 \mathrm{~kg} \mathrm{ha}^{-1}$ de $\mathrm{K}_{2} \mathrm{O}$ no solo com fertilidade baixa, e no solo com fertilidade média de $300 \mathrm{~kg} \mathrm{ha}^{-1}$ de N $190 \mathrm{~kg} \mathrm{ha}^{-1}$ de $\mathrm{P}_{2} \mathrm{O}_{5}$ e de $290 \mathrm{~kg} \mathrm{ha}^{-1}$ de $\mathrm{K}_{2} \mathrm{O}$, com produções máximas agronômicas de 13.083 e 10.468 $\mathrm{kg} \mathrm{ha}^{-1}$, nos solos com fertilidade baixa e média em P e K.

Esses valores de nutrientes também são bem maiores do que os recomendados para adubação de manutenção de gramíneas, para pasto exclusivo do grupo

Tabela 8 - Produção de matéria seca (MS) de forragem de Paspalum regnellii para as doses de nitrogênio interagindo com as de fósforo, em solos de fertilidade baixa e média, no segundo ano de estabelecimento.

\begin{tabular}{|c|c|c|c|c|c|c|c|c|}
\hline \multirow{3}{*}{$\begin{array}{l}\text { Doses de } \\
\mathrm{P}_{2} \mathrm{O}_{5} \\
\mathrm{~kg} \mathrm{ha}^{-1}\end{array}$} & \multicolumn{8}{|c|}{--------------------------------------- Doses de N ------------------------------------------- } \\
\hline & \multicolumn{8}{|c|}{------------ Solo fertilidade baixa -------- $\mathrm{kg} \mathrm{ha}^{-1}$} \\
\hline & 0 & 100 & 200 & 300 & 0 & 100 & 200 & 300 \\
\hline & \multicolumn{8}{|c|}{$\mathrm{kg} \mathrm{ha}^{-1} \mathrm{MS}$} \\
\hline 0 & 5.693 & 5.077 & 5.433 & 3.445 & 4.607 & 6.737 & 7.577 & 8.832 \\
\hline 70 & 5.882 & 7.473 & 9.985 & 7.350 & 4.912 & 7.059 & 7.961 & 9.321 \\
\hline 140 & 5.833 & 7.084 & 8.167 & 11.036 & 5.973 & 7.213 & 6.417 & 9.056 \\
\hline 210 & 6.828 & 9.285 & 11.663 & 10.841 & 3.777 & 6.425 & 8.500 & 9.146 \\
\hline
\end{tabular}


II, no Estado de São Paulo, grupo no qual o gênero Paspalum está incluído, e cujas doses máximas para solos com fertilidade baixa são: 60, 40 e 40 e fertilidade média: 60 , 20 e $20 \mathrm{~kg} \mathrm{ha}^{-1}$ de $\mathrm{N}, \mathrm{P}_{2} \mathrm{O}_{5}$ e $\mathrm{K}_{2} \mathrm{O}$, respectivamente (WERNER et al., 1996). Deve-se considerar que esses valores são recomendados para as forrageiras manejadas em sistema extensivo de pastejo. No sistema intensivo, onde é necessário obter altas produções de forragem, os nutrientes devem estar presentes em quantidade adequadas, pois qualquer nutriente pode limitar a produtividade das pastagens (SANTOS JÚNIOR, 2001).

Verificou-se que as produções de forragem de $P$. regnellii adubado foram semelhantes aos de Brachiaria decumbens intensamente adubada, quando se consideram quatro períodos de corte da pastagem estabelecida, e que variou de 9,8 a 13,5 $\mathrm{Mg}$ ha $^{-1}$ de matéria seca (PRIMAVESI et al., 2004). Quanto à qualidade de forragem, Batista et al. (2005) verificaram similaridade nos teores de proteína bruta e de fibra em detergente neutro com relação aos do capimbraquiária produzida em condições similares de solo e de fertilidade, podendo o teor de fibra ser menor quando se reduz a idade de corte para em torno de 20 dias, como verificado para $P$. atratum (HARE et al., 2001), cuja viabilidade necessita ser testada em nossas condições.

\section{CONCLUSÕES}

No ano de formação da pastagem de Paspalum regnellii, e no segundo ano, ocorrem respostas para nitrogênio e fósforo no solo com fertilidade baixa e para nitrogênio no solo com fertilidade média em fósforo e potássio.

A produção de forragem de $P$. regnellii é maior no primeiro ano no solo com fertilidade média em fósforo e potássio e, no segundo ano, é levemente superior no solo com fertilidade baixa.

\section{REFERÊNCIAS BIBLIOGRÁFICAS}

ANDRADE, D. F.; NOLETO, A. Q. Exemplos de fatoriais fracionados $(1 / 2) 4^{3}$ e $(1 / 4)^{4}$ para o ajuste de modelos polinomiais quadráticos. Pesquisa Agropecuária Brasileira, Brasília, v. 21, p. 677-680, 1986.

BATISTA, L. A. R.; GODOY, R. Caracterização preliminar e seleção de germoplasma do gênero Paspalum para produção de forragem. Revista Brasileira de Zootecnia, Viçosa, v. 29, n. 1, p. 23-32, 2000.

BATISTA, L. A. R.; MEIRELLES, P. R. de L.; GODOY, R. Produção e qualidade da forragem em acessos selecionados de Paspalum na região central do estado de
São Paulo. In: REUNIÃO ANUAL DA SOCIEDADE BRASILEIRA DE ZOOTECNIA, 42., 2005, Goiânia, Goiás. Anais... Goiânia: UFGO, 2005.

BRÂNCIO, P. A.; NASCIMENTO JÚNIOR, D.; EUCLIDES, V. P. B.; REGAZZI, A. J.; ALMEIDA, R. G.; FONSECA, D. M.; BARBOSA, R. A. Avaliação de três cultivares de Panicum maximum Jacq. sob pastejo: composição química e digestibilidade da forragem. Revista Brasileira de Zootecnia, Viçosa, v. 31, n. 4, p. 1605-1613, 2002.

BUCHNER, A.; STURM, H. Gezielter duengen: intensiv, wirtschaftlich, umweltbezogen. Frankfurt-Main: LG Verlag, 1980. 319 p.

CANTARELLA, H. Adubação com nitrogênio, potássio e enxofre. In: RAIJ, B. van; CANTARELLA, H.; QUAGGIO, J. A.; FURLANI, A. M. C. (Eds.). Recomendações de adubação e calagem para o Estado de São Paulo. Campinas: Instituto Agronômico, 1996. p. 22-27. (Boletim técnico, 100).

COSTA, C.; FAVORETTO, V.; MALHEIROS, E. B. Variação na estrutura da vegetação de duas cultivares de Panicum maximum Jacq. (Colonião e Tobiatã) submetidas a diferentes tipos de manejo. Composição em proteína bruta e digestibilidade "in vitro" da matéria seca. Pesquisa Agropecuária Brasileira, Brasília, v. 27, n. 12, p. 16591670, 1992.

CUNHA, M. K.; SIEWERDT, L.; SILVEIRA JÚNIOR, P.; SIEWERDT, F. Doses de nitrogênio e enxofre na produção e qualidade da forragem de campo natural de planossolo no Rio Grande do Sul. Revista Brasileira de Zootecnia, Viçosa, v. 30, n. 3, p. 651-658, 2001.

HARE, M. D.; SAEGKHAM, M.; KAEWKUNYA, C.; TUDSRI, S.; SURIYAJANTRATONG, W.; THUMMASAEG, K.; WONGPICHET, K. Effect of cutting on yield and quality of Paspalum atratum in Thailand. Tropical Grasslands, Brisbane, v. 35, p. 144-150, 2001.

KALMBACHER, R. S.; MARTIN, F. G. Effect of N rate and time of application on atra paspalum. Tropical Grasslands, Saint Lucia, v. 33, p. 214-221, 1999.

MERTENS, D. R. Regulation of forage intake. In: FAHEY JUNIOR, G. C.; COLLINS, M.; MERTENS, D. R.; MOSER, L. E. (Eds.). Forage quality, evaluation and utilization. Madison: ASA; CSSA; SSSA, 1994. cap. 11, p. 450-493. 
MILFORD, R.; MINSON, D. J. Intake of tropical pasture species. In: CONGRESSO INTERNACIONAL DE PASTAGENS, 9., 1965, São Paulo. Anais... São Paulo: USP, 1965. v. 1, p. 815-822.

NOLLER, C. H.; RHYKERD, C. L. Relation of nitrogen fertilization and chemical composition of forage to animal health and performance. In: MAYS, D. A. Forage fertilization. Madison: Crop Science Society of America, 1974. cap. 17, p. 363-393.

OLIVEIRA, P. P. A.; BOARETTO, A. E.; TRIVELIN, P. C. O.; OLIVEIRA, W. S.; CORSI, M. Liming and fertilization for restoring degraded Brachiaria decumbens pasture on sandy soil. Scientia Agricola, Piracicaba, v. 60, n. 1, p. 125-131, 2003.

OLIVEIRA, P. P. A.; TRIVELIN, P. C. O.; OLIVEIRA, W. S.; CORSI, M. Fertilização com $\mathrm{N}$ e $\mathrm{S}$ na recuperação de pastagem de Brachiaria brizantha cv. Marandu em Neossolo Quartzarênico. Revista Brasileira de Zootecnia, Viçosa, v. 34, n. 4, p. 1121-1129, 2005.

PRIMAVESI, O.; CORRÊA, L. A.; PRIMAVESI, A. C.; CANTARELLA, H.;ARMELIN, M. J.A.; SILVA,A. G; FREITAS, A. R. de. Adubação com uréia em pastagem de Cynodon dactylon cv. Coastcross: eficiência e perdas. São Carlos: Embrapa Pecuária Sudeste, 2001. 42 p. (Circular técnica, 30).

PRIMAVESI, O.; CORRÊA, L. A.; PRIMAVESI, A. C.; CANTARELLA, H.; SILVA, A. G. Adubação com uréia em pastagem de Brachiaria brizantha sob manejo rotacionado: eficiência e perdas. São Carlos: Embrapa Pecuária Sudeste, 2003. 6 p. (Comunicado técnico, 41).

PRIMAVESI, O.; PRIMAVESI, A. C.; CORRÊA, L. A.; ARMELIN, M. J. A.; FREITAS, A. R. Calagem em pastagem de Brachiaria decumbens recuperada com adubação nitrogenada em cobertura. São Carlos: Embrapa Pecuária Sudeste, 2004. 32 p. (Circular técnica, 37).

RAIJ, B. van; QUAGGIO, J. A.; CANTARELLA, H.; ABREU, C. A. Interpretação de resultados de análise de solo. In: RAIJ, B. van; CANTARELLA, H.; QUAGGIO, J. A.; FURLANI, A. M. C. (Eds.). Recomendações de adubação e calagem para o Estado de São Paulo. Campinas: Instituto Agronômico, 1996. p. 8-13. (Boletim técnico, 100).

RAIJ, B. van; ANDRADE, J. C.; CANTARELLA, H.; QUAGGIO, J. A. (Eds.). Análise química para avaliação da fertilidade de solos tropicais. Campinas: IAC, 2001. 285 p.
REID, R. L.; JUNG, G. S.; THAYNE, W. V. Relationships between nutritive quality and fiber components of cool season and wam season forages: a retrospective study. Journal of Animal Science, Champaign, v. 66, p. 12751291, 1988.

RUGGIERI, A. C.; FAVORETTO, V.; MALHEIROS, E. B. Efeito de níveis de nitrogênio e regimes de corte na distribuição, na composição bromatológica e na digestibilidade "in vitro" da matéria seca da Brachiaria brizantha (Hochst) Stapf cv. Marandu. Revista Brasileira de Zootecnia, Viçosa, v. 24, n. 2, p. 222-232, 1995.

SANDLES, L. Forage utilization efficiency: an Australian perspective. In: LYONS, T. P.; JACQUES, K. A. (Eds.). Biotechnology in the feed industry. Nottingham: Altech, 1999. p. 343-354.

SANTOS JÚNIOR, J. D. G. Dinâmica de crescimento e nutrição do capim-marandu submetido a doses de nitrogênio. 2001. 79 p. Dissertação (Mestrado em Agronomia) - Escola Superior de Agricultura "Luiz de Queiroz”, Piracicaba, 2001.

SAS INSTITUTE. SAS/STAT user's guide: statistics. Versão 6.4. Cary, 1993. 1686 p.

SILVA, D. J. Análise de alimentos, métodos químicos e biológicos. Viçosa: UFV, 1981. 166 p.

SILVA, M. M. P.; VASQUEZ, H. M.; SILVA, J. F. C.; BRESSAN-SMITH, R. E.; ERBESDOBLER, E. A.; SOARES, C. S. Composição bromatológica, disponibilidade de Fforragem e índice de área foliar de 17 genótipos de capim-elefante (Pennisetum purpureum Schum.) sob pastejo, em Campos dos Goytacazes, RJ. Revista Brasileira de Zootecnia, Viçosa, v. 31, n. 1, p. 313-320, 2002.

SOUZA, G. B. de; NOGUEIRA, A. R. de; BATISTA, L. A. R. Método alternativo para determinação de fibra em detergente neutro e detergente ácido. São Carlos: Embrapa Pecuária Sudeste, 1999. 21 p. (Boletim de pesquisa, 4).

WERNER, J. C.; PAULINO, V. T.; CANTARELLA, H. Forrageiras. In: RAIJ, B. van; CANTARELLA, H.; QUAGgiO, J. A.; FURLANI, A. M. C. (Eds.). Recomendações de adubação e calagem para o Estado de São Paulo. Campinas: Instituto Agronômico, 1996. p. 263 273. (Boletim técnico, 100). 